\title{
Human Neutrophil Peptides 1-3 - Early markers in development of colorectal adenomas and carcinomas
}

\author{
Henning Mothes ${ }^{\mathrm{a}}$, Christian Melle ${ }^{\mathrm{b}}$, Günther Ernst ${ }^{\mathrm{b}}$, Roland Kaufmann ${ }^{\mathrm{a}}$, Ferdinand von Eggeling ${ }^{\mathrm{b}}$ and \\ Utz Settmacher ${ }^{\text {a,* }}$ \\ ${ }^{a}$ Department of General, Visceral and Vascular Surgery, Universitätsklinikum Jena, Jena, Germany \\ ${ }^{\mathrm{b}}$ Core Unit Chip Application (CUCA), Institute of Human Genetics and Anthropology, Theoretikum, Jena, Germany
}

\begin{abstract}
Expression of Human Neutrophil Peptides (HNP) 1-3 was recently found to be associated with development of colorectal cancer. Raised defensin-expression in tumours is believed to stem from increased infiltration of neutrophils into tumour environment.

To further specify the role of $\alpha$-defensins in tumourigenesis and progression, HNP1-3 were analyzed in colorectal adenomas and carcinomas of 87 patients and quantified in relation to cancer stage and grading. Using the ProteinChip arrays, HNP1-3 were found upregulated in both colorectal adenomas and carcinomas. By combining the array with Laser capture microscopy we were able to confirm that HNP1-3 are expressed by tumour cells but not by neutrophils or other tumour invading cells. These findings suggest that $\alpha$-defensins are more likely to contribute to tumour growth than they are to mount an effective host anti-tumour response. However, the amount of HNP-expression was not found to be related to tumour stage, grading, and serological tumour markers.
\end{abstract}

Keywords: ProteinChip Array, SELDI, tissue microdissection, Human Neutrophil Peptides, colorectal adenoma, colorectal carcinoma

\section{Introduction}

It is known that the intestinal mucosa is not only a physical barrier but also acts as part of the innate and adaptive immune system. Antimicrobial and chemotactic peptides, including defensins, seem to be key components of this mucosal immunity [19]. The widespread expression of defensins in epithelial cells of the gastrointestinal tract suggests that they play an important role in the maintenance of a stable cell- and microbial population. This includes preventing invasion of host tissues by luminal bacteria and ingested pathogens [7].

*Correspondening author: Prof. Dr. U. Settmacher, Department for General, Visceral and Vascular Surgery, University Hospital Jena, Erlanger Allee 101, 07747 Jena, Germany. Tel.: +49 36419322601 ; Fax: +493641 9322602; E-mail: utz.settmacher@med.uni-jena.de.
HNP1-3 are part of the $\alpha$-defensin family of peptides, which in humans include six known members. They are normally synthesized in neutrophil precursor cells, and released by mature circulating neutrophils at inflammatory sites. Raised HNP1-3 expression from intestinal epithelial cells was also observed in active inflammatory bowel disease [8]. Furthermore, two recent studies confirmed, that colorectal tumorigenesis was proven to be associated with the regulation of $\alpha$ defensins in vivo $[1,2,13]$. Development of colorectal carcinomas (CRC) and adenomas is known to be associated with chronic mucosal inflammation and exposure to dietary carcinogens. However, most of the adenomas do not proceed to undergo further malignant transformation suggesting that the risk of tumour development is determined by both genetic and environmental factors. The impact of the mutation of the $A p c$ gene on defensin gene expression through activation of 
the $\beta$-catenin signaling pathway highlights this association [3].

In previous tests we already identified HNP1-3 with significant different expression patterns between normal colonic mucosa and carcinoma using ProteinChip technology [13]. The ProteinChip Array was used as a screening method of protein lysats of tumour tissue and normal mucosa, where in the low range (2-20 kilodaltons), more than 100 peaks were detected with normalized intensities. Three peak masses between 3.40 and 3.50 kilodaltons with remarkably low $P$ values were selected for further characterization and identified as Human Neutrophil Peptides (HNP) 1-3 by immunodepletion [13]. To ensure that the localized HNP1-3 are identical to the peaks found by ProteinChip analysis, IHC-positive and -negative cell areas were obtained by tissue laser microdissection in previous tests [13]. In protein lysates from the positive fraction, signals identical in mass to the peaks obtained with the initial ProteinChip analysis were detected. In the protein lysate from the negative fraction, these peaks were not visible.

Based on our earlier results, the objectives of this study were (1) to measure HNP expression also in colonic adenomas and (2) to examine the role of $\alpha$ defensins in the clinical progression of CRC.

\section{Materials and methods}

\subsection{Laser microdissection of tissue sections}

Experiments and collection of tissue samples have been approved by the ethical committee of the University of Jena. Ninety-nine samples of colorectal adenomas, carcinomas, and normal colonic mucosa were obtained following colorectal surgery or endoscopy and snap frozen in liquid nitrogen immediately after resection, and stored at $-80^{\circ} \mathrm{C}$. Tumour specimens were categorized according to UICC- and WHO classification. Laser microdissection was performed with a Laser Microdissection and Pressure Catapulting Microscope (LMPC; Palm, Bernried, Germany) as described elsewhere [11]. Approximately 3000 to 5000 cells from native air-dried cryostat tissue sections were microdissected within 20 minutes, excluding inflammatory areas within the tumour. Proteins were extracted by a lysis buffer $(100 \mathrm{mmol} / \mathrm{L} \mathrm{Na}$-phosphate $(\mathrm{pH}$ 7.5), $5 \mathrm{mmol} / \mathrm{L}$ EDTA, $2 \mathrm{mmol} / \mathrm{L} \mathrm{MgCl} 2,3 \mathrm{mmol} / \mathrm{L}$ 2- $\beta$-mercaptoethanol, $0.1 \%$ CHAPS, $500 \mu \mathrm{mol} / \mathrm{L}$ leupeptin, and $0.1 \mathrm{mmol} / \mathrm{L}$ PMSF) for 30 minutes on ice. After centrifugation (15 minutes; $15.000 \mathrm{rpm}$ ), the supernatant was immediately analyzed or frozen in liquid nitrogen for a maximum of 1 day.

\subsection{Profiling of microdissected normal colon epithelium, adenoma and carcinoma tissue}

The protein lysates from microdissected tissues (tumour, adenoma and normal) were analyzed on both strong anion exchange arrays (SAX2) and weak cation exchange arrays (WCX2; Ciphergen Biosystems Inc, Fremont, CA) as described elsewhere [11]. Array spots were preincubated by a washing/loading buffer containing $100 \mathrm{mmol} / \mathrm{L}$ Tris-buffer, $\mathrm{pH} 8.5$, with $0.02 \%$ Triton $\mathrm{X}-100$ for SAX arrays and $100 \mathrm{mmol} / \mathrm{L}$ Tris-buffer, $\mathrm{pH}$ 4.5 , with $0.02 \%$ Triton X-100 for WCX arrays followed by application of $2 \mu \mathrm{L}$ sample extract on ProteinChip arrays, which were incubated at room temperature for 90 minutes in a humidity chamber. After washing 3 times with the same buffers and 2 final washing steps with water, $2 \times 0.5 \mu \mathrm{L}$ sinapinic acid (saturated solution in $0.5 \%$ TFA $/ 50 \%$ acetonitrile) was applied. Mass analysis was performed in a ProteinChip Reader (PBS II, Ciphergen Biosystems Inc, Fremont, CA) according to an automated data collection protocol. Cluster analysis of the detected signals and the determination of respective $P$ values for normal and tumor tissue were carried out with the Biomarker Wizard Program (version 3.0; Ciphergen Biosystems Inc). For Pvalue calculation, spectra with at least 10 signals in the range between 2 and 20 kilodaltons exhibiting a signal-to-noise $(\mathrm{S} / \mathrm{N})$ ratio of at least 5 were selected and analyzed with the Mann-Whitney $U$ test for nonparametric data sets.

\subsection{Characterization of proteins by immunohistochemistry}

Eight-micrometer cryostat sections of colon cancer and adenoma tissue and normal colon epithelium were placed on slides, air dried for 60 minutes at $20^{\circ} \mathrm{C}$, and fixed in paraformaldehyde as described previously [11]. After fixation, slides were treated in the microwave at 80 watts $(3 \times 3$ minutes $)$ in $10 \mathrm{mmol} / \mathrm{L}$ citric acid, $\mathrm{pH}$ 6.0 , to inhibit endogenous peroxidatic activity. Subsequently, they were rinsed twice with TBS, $\mathrm{pH} 7.4$, and incubated overnight at $4{ }^{\circ} \mathrm{C}$ in a humidity chamber with the corresponding primary monoclonal antibody against $\alpha$-defensins 1-3 (T-1034; BMA Biomedicals; Augst, Switzerland). Slides were rinsed $3 \times 10$ minutes in TBS and incubated at room temperature for 40 minutes with the secondary biotinylated monoclonal antibody (Santa Cruz Biotechnology, Santa Cruz, CA). Slides were rinsed $3 \times 10$ minutes in TBS and the Vectastain Elite ABC kit (Vector Laboratories, Burlingame, $\mathrm{CA}$ ) and the Jenchrom pxbl-kit (MoBiTec, Göttingen, 
Germany) were used according to the manufacturer's instructions to visualize antibody localization. Negative controls were incubated with the labelled secondary antibody only. Sections cut in parallel to the IHC-treated sections were stained by H\&E for better identification of different tissue areas. IHC staining was evaluated by a Pathologist and an Anatomist.

The 1-photon laser-scanning microscopy was performed with a LSM 310 (Carl Zeiss, Oberkochen-Jena, Germany) in the transmission mode using an Argonion laser at a wavelength of $480 \mathrm{~nm}$. In most cases, Zeiss objective NEOFLUAR 40X/1.30 oil was used at a scanning time of 60 seconds [9].

\subsection{Evaluation of clinical and serological parameters}

Serum was collected from patients following informed consent on the morning of their operation at 7 am; the patients were all starved from midnight, and whilst lying in bed had already received $1000 \mathrm{ml}$ of normal saline overnight. One serum tube was sent to the lab for centrifugation within 6 hours and was then snap frozen in liquid nitrogen. Another serum tube was sent to the hospital lab for evaluation of full white blood count, C-reactive protein and the gastrointestinal tumour markers carcinoembryogenic antigen (CEA) and CA19-9. Clinical and serological parameters were then collected and prepared for statistical analysis.

\subsection{Statistical analysis}

Statistical analysis was performed using the SPSS software package. For analysis of the quantitative parameters, the T-Test was used if normal distribution could be confirmed by Kolmogoroff-Smirnow test. The Mann Whithney test was used for comparison of quantitative parameters with tumour stages and grading. The direct correlation between quantitative parameters was analyzed using the Pearson's test for dependent variables. P-values of less than 0.05 were considered as indicating a significant difference.

\section{Results}

\subsection{Microdissection and protein profiling of differential expressed protein peaks}

For our study, areas corresponding to approximately 3000 to 5000 cells per tissue probe were excised, and 65 tissue sections ( 29 adenoma and 36 normal colonic

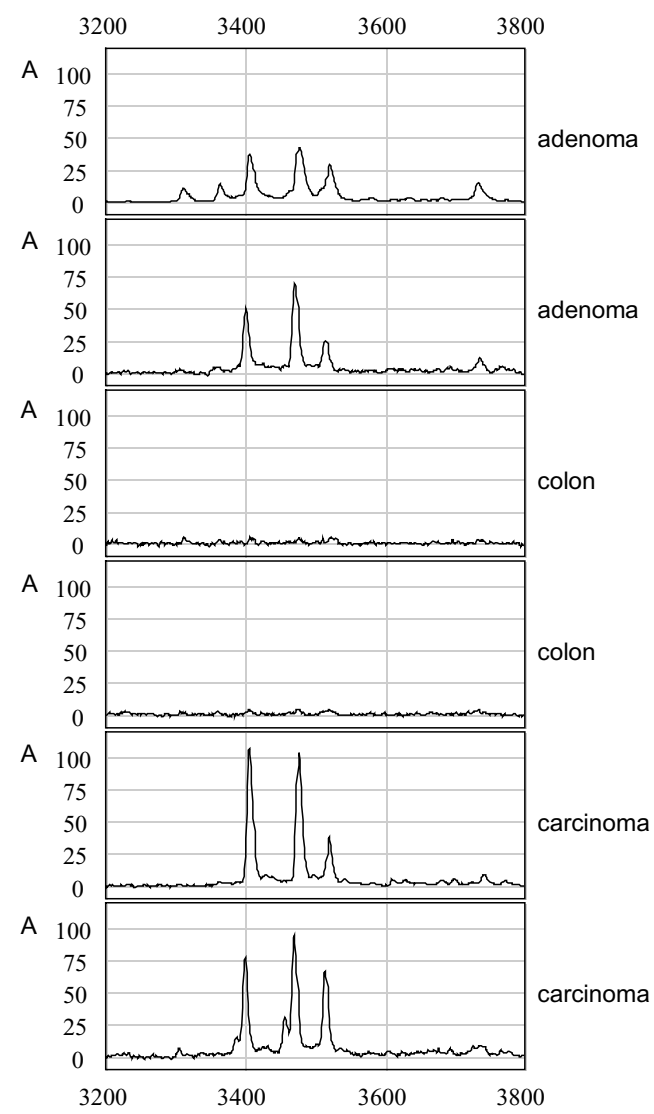

Fig. 1. Representative examples of SELDI-TOF MS spectra of colon adenoma, CRC and normal colon epithelium. Data are obtained using SAX2 array. The three peaks correspond to HNP 2 (3.4 kDA), HNP 1 (3.47 kDa) and HNP 3 (3.5 kDa).

epithelium tissues) dissected. All protein lysates from the microdissected tissues were applied to SAX2 and WCX arrays and analyzed on a ProteinChip Reader and compared to the profiles of 34 colon cancer tissue samples, which were previously examined, with the same method [13]. After evaluation with the Biomarker Wizard Program (Ciphergen Biosystems Inc), three peak masses (Fig. 1) of approximately $3.40(P=6.9$ $\left.\times 10^{-6}\right), 3.47\left(P=2.62 \times 10^{-5}\right)$, and 3.50 kilodaltons $\left(P=2.04 \times 10^{-4}\right)$ that possessed remarkably low $P$ values were identified as Human Neutrophil Peptides (HNP) 1-3.

\subsection{Localization of HNP 1-3 by immunohistochemistry}

To confirm identification of HNP1-3 and, above all, to localize HNP1-3 in tissue sections, we examined their expression in 10 different colon cancer tissues, 

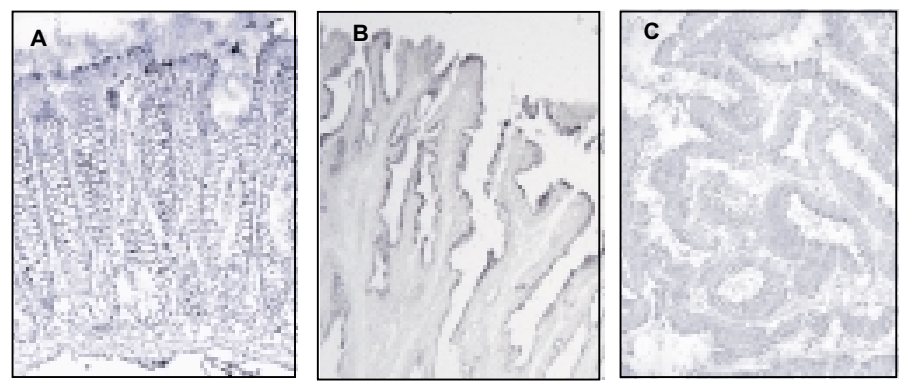

Fig. 2. Immunohistochemistry (IHC) of HNP1-3 visualized by normal microscopy. Cytoplasmatic HNP1-3 staining of epithelial cells is preferably seen in apical areas. Normal colonic mucosa shows weak intensity (A, magnification of 80x); colonic adenoma (B, 80x) and carcinoma (C, 120x) exhibit a strong positive reaction.

adenomas and adjacent normal tissue sections using immunohistochemistry (IHC). Normal colonic epithelium showed a weak positive reaction (Fig. 2A). On the other hand, tumour tissue (adenoma and carcinoma) displayed an explicitly strong reaction (Fig. 2B and 2C). Negative controls without the primary or with no antibody at all demonstrated negative results. A positive stain for HNP1-3 could also be found in fibroblasts and macrophages of the mucous membrane.

\subsection{Quantification of proteins; clinical and serological parameters}

87 patients were included in this study with a mean age of 66.8 (44-92) years. 44 patients were female $(51 \%)$. Defensin-expression was found to be independent from age and sex of the patients. Correlation between HNP 1, 2, and 3 was constant throughout all tests.

The characteristic signals between 3.40 and 3.50 $\mathrm{kDa}$ showed an increased expression in samples derived from colorectal adenomas $\left(P=2.6 \times 10^{-2}\right)$ and carcinomas $\left(P=6.3 \times 10^{-6}\right)$ as compared to normal colonic mucosa; also significantly discriminating between both $\mathrm{CRC}$ and adenoma tissue $\left(P=1.6 \times 10^{-4}\right)$ respectively. The distribution of the intensities for the different tissues is shown in Fig. 3. Representative examples of SELDI-MS spectra from colorectal adenoma, carcinoma, and normal mucosa are given in Fig. 1 ranging between 3.2 and $3.8 \mathrm{kDa}$. Further characteristic peaks have been found and corresponding proteins identified as described elsewhere [10,12].

To correlate clinical data with protein profiles, a group of 39 previously examined patients with CRC was specified, in which clinical and serological data were available. As described earlier, expression of HNP1-3 was higher in CRC cells (median 9.98 $\mathrm{ng} / \mathrm{ml}$ (standard deviation 14.11) as compared to nor- mal colonic mucosa $(0.70 \mathrm{ng} / \mathrm{ml}(1.96)$ as analyzed by a specific ELISA [13]. The quantities of HNP1, 2, and 3 showed a strong correlation to each other. Results were crosschecked and found similar for all stages of the UICC-classification (Table 1). Using the MannWhithney-U-Test, expression of defensins derived from tumour was then compared to TNM staging and grading. Taking HNP1 as a representative for all three neutrophil peptides, the values did not show any statistical significance in relation to invasiveness of the tumour (T), lymph node involvement $(\mathrm{N})$, metastatic disease (M), and grading $(\mathrm{G})$ (Table 1). In addition, CEA, CA19-9, CRP and WBC levels in serum were correlated with the expression of HNP1-3 in CRC tissues, but no correlation could be determined with $P$-values of 0.83 for CEA, 0.13 for CA19-9, 0.68 for CRP and 0.25 for the white blood count.

\section{Discussion}

Based on our earlier results, we now evaluated whether HNP expression is already raised in colonic adenomas, which might highlight its relation to early tumorigenesis. In a recent study, elevated concentrations of HNP1-3 were found in colorectal cancer compared to normal colonic tissue [1]. These results were based on SELDI-TOF/MS, the same technique used in our study. However, tissue screening was performed by comparing protein lysates of tissue samples without microdissection. It could therefore, not be determined whether the peptides were produced by colon cancer cells or by tumour infiltrating neutrophils [1].

The additional use of microdissection prior to tumour analysis is essential to separate epithelial or mesenchymal tumour cells from tumour stroma, pre-existent tissue components, necrotic and apoptotic areas, or areas with inflammation [17]. Thus, the lack of proteins from 


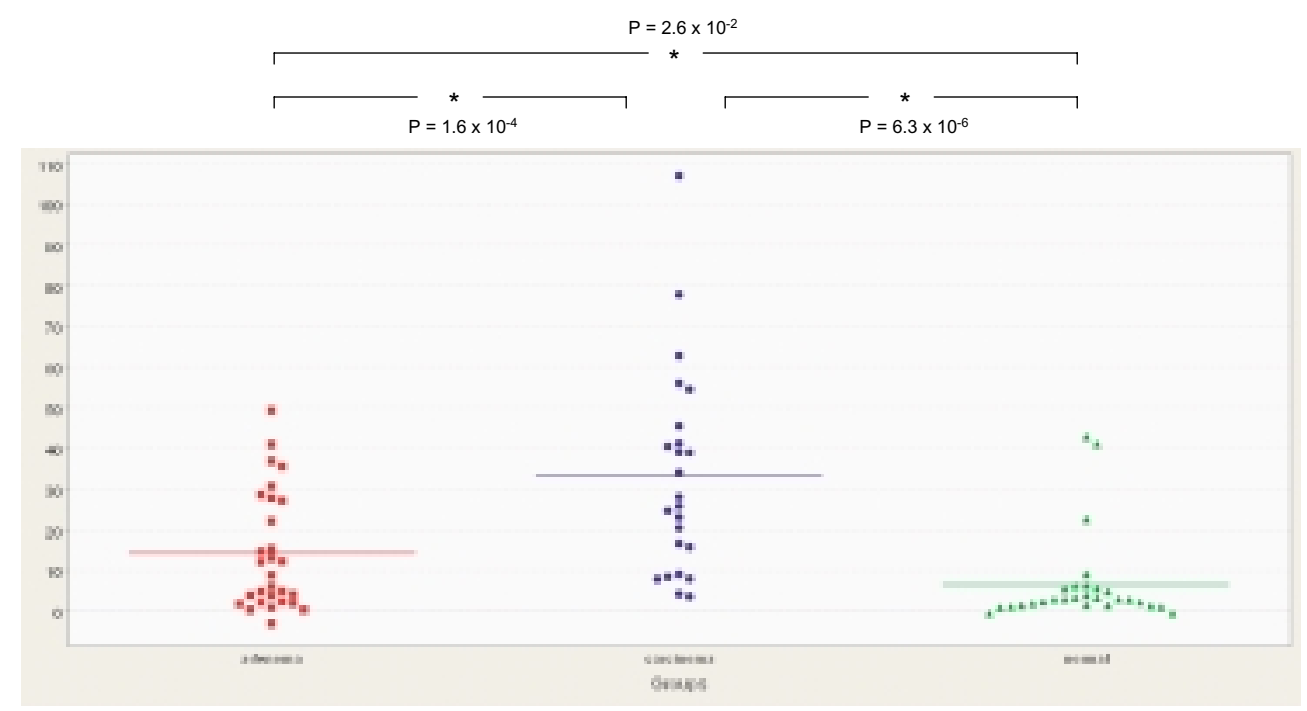

Fig. 3. The distribution of the intensities of 3,47 $\mathrm{kDa}$ peak in colon adenoma samples (adenoma), CRC samples (carcinoma) and normal colon epithelium samples (normal). The spectra were obtained using SAX2 arrays. X-axis indicates the sample groups, Y-axis the intensity ( $\mu \mathrm{A}$ ).

Table 1

The mean value and SD ( $\mathrm{ng} / \mathrm{ml})$ of HNP1 concentrations in extracts of colon cancer tissue in relation to tumor stage according to the UICC- and TNM-classifications and grading (ELISA-test). No significant differences found in relation to the clinical progress of the tumour.

\begin{tabular}{ccc}
\hline & $\mathrm{n}$ & HNP 1 \\
\hline UICC classification & 7 & $21.24(17.97)$ \\
stage 1 & 12 & $6.58(11.38)$ \\
stage 2 & 6 & $4.19(8.39)$ \\
stage 3 & 8 & $17.17(15.49)$ \\
stage 4 & & \\
Invasiveness & 1 & 17.2 \\
$\mathrm{~T}_{1}$ & 8 & $3.67(15.56)$ \\
$\mathrm{T}_{2}$ & 19 & $11.46(11.35$ \\
$\mathrm{T}_{3}$ & 5 & $8.22(21.12)$ \\
$\mathrm{T}_{4}$ & & \\
Lymph node involvement & 20 & $11.46(15.0)$ \\
$\mathrm{N}_{0}$ & 7 & $3.24(15.52)$ \\
$\mathrm{N}_{1}$ & 5 & $9.98(14.02)$ \\
$\mathrm{N}_{2}$ & 1 & 12.86 \\
$\mathrm{~N}_{3}$ & & \\
Metastases & 25 & $8.12(14.25)$ \\
$\mathrm{M}_{0}$ & 8 & $17.17(14.92)$ \\
$\mathrm{M}_{1}$ & & \\
$\mathrm{G}_{2}$ & 22 & $9.98(16.2)$ \\
$\mathrm{G}_{3}$ & 11 & $11.31(10.83)$ \\
\hline Grading & &
\end{tabular}

other contaminating tissues assures a high degree of reproducibility, because inhomogeneity is eliminated during the collection step [6]. Therefore, microdissection offers the possibility of reducing the complexity of the proteome by using a defined cell population [17]. Its compatibility with ProteinChip technology has been shown in different studies $[5,15,16,18]$. However, for the analysis of proteins from tumour tissue, two problems needed to be solved: firstly, the tissue heterogeneity of samples, and secondly, the heterogeneity of the tumour itself. An adequate solution could be found for the first problem separating epithelial tissue from connective tissue with LMPC. Tumour heterogeneity in respect to transscriptomes and proteomes is morphologically difficult to recognize and therefore cannot be completely deciphered by microdissection [11].

With our results, it could be clarified for the first time, that HNP1-3 are expressed in colon carcinoma and adenoma cells and not by infiltrating neutrophils. To further specify how this expression was associated with cancer development, we correlated HNP expression with UICC classification, TNM stages, and grading of the tumour. HNP1-3 were raised in all CRC compared to normal colonic mucosa and adenomas without showing differences according to tumour stage and grading. This is in agreement with the results of a recent study [2], where increased expression of HNP1-3 in CRC tissue was not found to be related to Dukes' classification. It seems that HNP1-3 are already raised in early tumour stages in comparison with normal colonic mucosa, but then remain on this level, without increasing according to the malignant potential of the tumour.

Therefore the question was raised whether HNP1-3 is already found elevated in pre-malignant lesions of the colon. Indeed, defensins were also expressed at a higher rate in adenomas as compared to normal mucosa, but to a lesser extent than in cancer tissue. These results, 
together with the histological evidence that HNP1-3 are expressed in CRC cells and not in surrounding tissue, make the association between HNP-expression and CRC development highly probable.

Since $\alpha$-defensins are known as peptides with antimicrobial and chemotactic function, we firstly examined a possible relation between HNP expression and (tumour related) local and systemic inflammation. Firstly, our histological sections did not show higher invasion of neutrophils in relation to tumour stage (data not shown). Furthermore, apart from a weak correlation between C-reactive protein (CRP) and expression of HNP1 and HNP2 in normal colonic mucosa, leucocytes and CRP did not show any association with the stage of CRC according to the UICC classification. However, it was not the aim of this study to fully exclude any correlation between defensin-expression of tumour cells with any inflammation within the tumour environment. Further investigations regarding this topic are currently under progress.

Our results suggest that HNP1-3 are abundant in adenomas and CRC. In active inflammatory bowel disease, where HNP1-3 were also found to be expressed in high amounts, it is not clear whether epithelial expression of HNP1-3 is induced by the inflammatory state or whether the peptides are released by adjacent neutrophils and taken up by the epithelial cells [8]. Here it is believed that the peptides provide protection against microbial invasion in response to damage of the mucosal barrier. HNP1-3 are known to stimulate epithelial cells of the bronchial wall to upregulate interleukin-8 production [14], a potent neutrophil chemotactic factor. Thus, it was suggested that upregulated expression of HNP1-3 in tumours may primarily originate from invading immune cells, but could be initiated by HNP1-3 producing cancer cells [1]. However, since HNP1-3 were also found to be elevated in adenomas, this hypothesis was not supported by our results. We rather assume that HNP1-3 are not yet known members of an inflammatory peptide network [4] within tumours that is known to regulate communication between tumour and stroma cells and therefore influence cell survival, proliferation, and differentiation.

\section{Acknowledgement}

This work was supported by a grant of the German Federal Ministry of Education and Research (BMBF) and the Interdisciplinary Center for Clinical Research (ICCR), Jena. We thank Annette Bleul and Bettina Schimmel for their technical support and Dr. Heike Hoyer for assisting in statistical analysis of the data.

\section{References}

[1] J. Albrethsen, R. Bogebo, S. Gammeltoft, J. Olsen, B. Winther and H. Raskov, Upregulated expression of human neutrophil peptides 1, 2 and 3 (HNP 1-3) in colon cancer serum and tumours: a biomarker study, BMC Cancer 5(1) (2005), 8.

[2] J. Albrethsen, C.H. Moller, J. Olsen, H. Raskov and S. Gammeltoft, Human neutrophil peptides 1, 2, and 3 are biochemical markers for metastatic colorectal cancer, Eur J Cancer 42(17) (2006), 3057-3064.

[3] P. Andreu, S. Colnot, C. Godard et al., Crypt-restricted proliferation and commitment to the Paneth cell lineage following Apc loss in the mouse intestine, Development 132 (2005), 1443-1451.

[4] F. Balkwill and A. Mantovani, Inflammation and cancer: back to Virchow? Lancet 357 (2001), 539-545.

[5] C.H. Cazares, B.L. Adam, M.D. Ward et al., Normal, benign, preneoplastic, and malignant prostate cells have distinct protein expression profiles resolved by surface enhanced laser desorption/ionization mass spectrometry, Clin Cancer Res $\mathbf{8}$ (2002), 2541-2552.

[6] R.A. Craven and R.E. Banks, Laser capture microdissection and proteomics: possibilities and limitation, Proteomics 1(10) (2001), 1200-1204.

[7] R.N. Cunliffe and Y.R. Mahida, Expression and regulation of antimicrobial peptides in the gastrointestinal tract, J Leukoc Biol 75 (2004), 49-58.

[8] R.N. Cunliffe, Alpha-defensins in the gastrointestinal tract, Mol Immunol 40(7) (2003), 463-467.

[9] K.J. Halbhuber and K. König, Modern laser scanning microscopy in biology, biotechnology and medicine, Annals of Anatomy-Anatomischer Anzeiger 185 (2003), 1-20.

[10] C. Melle, R. Bogumil, G. Ernst, B. Schimmel, A. Bleul and F. von Eggeling, Detection and identification of heat shock protein 10 as a biomarker in colorectal cancer by protein profiling, Proteomics 6(8) (2006), 2600-2608.

[11] C. Melle, G. Ernst, B. Schimmel et al., Biomarker discovery and identification in laser microdissected head and neck squamous cell carcinoma with ProteinChip Technology, Twodimensional Gel Electrophoresis, Tandem Mass Spectrometry, and Immunohistochemistry, Mol Cell Proteomics 2 (2003), $443-452$.

[12] C. Melle, G. Ernst, B. Schimmel et al., Different expression of calgizzarin (S100A11) in normal colonic epithelium, adenoma and colorectal carcinoma, Int J Oncol 28(1) (2006), 195-200.

[13] C. Melle, G. Ernst, B. Schimmel et al., Discovery and identification of alpha-defensins as low abundant, tumour-derived serum markers in colorectal cancer, Gastroenterology 129(1) (2005), 66-73.

[14] S. van Wetering, S.P. Mannesse-Lazeroms, M.A. van Sterkenburg, M.R. Daha, J.H. Dijkman and P.S. Hiemstra, Effect of defensins on interleukin-8 synthesis in airway epithelial cells, Am J Physiol 272 (1997), 888-896.

[15] F. von Eggeling, H. Davies, L. Lomas et al., Tissue-specific microdissection coupled with ProteinChip array technologies: Applications in cancer research, Biotechniques 29 (2000), 1066-1070.

[16] F. von Eggeling, K. Junker, W. Fiedler et al., Mass spectrometry meets chip technology: A new proteomic tool in cancer research? Electrophoresis 22 (2001), 2898-2902.

[17] F. von Eggeling, C. Melle and G. Ernst, Biomarker discovery by tissue microdissection and ProteinChip Array analysis, $J$ Lab Med 27 (2003), 1-6. 
[18] G.L. Wright, L.H. Cazares, S.M. Leung et al., ProteinChip surface enhanced laser desorption/ionization (SELDI) mass spectrometry: a novel protein biochip technology for detection of prostate cancer biomarkers in complex protein mixtures,
Prostate Cancer Prostatic Dis 2 (1999), 264-276.

[19] D. Yang, Q. Chen, O. Chertov, J.J. Oppenheim, Human neutrophil defensins selectively chemoattract native $\mathrm{T}$ and immature dendritic cells, J Leukocyte Biol 68 (2000), 9-14. 


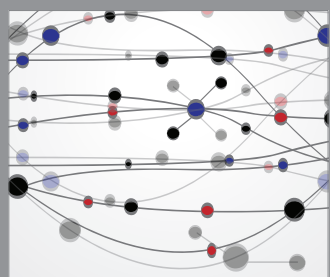

The Scientific World Journal
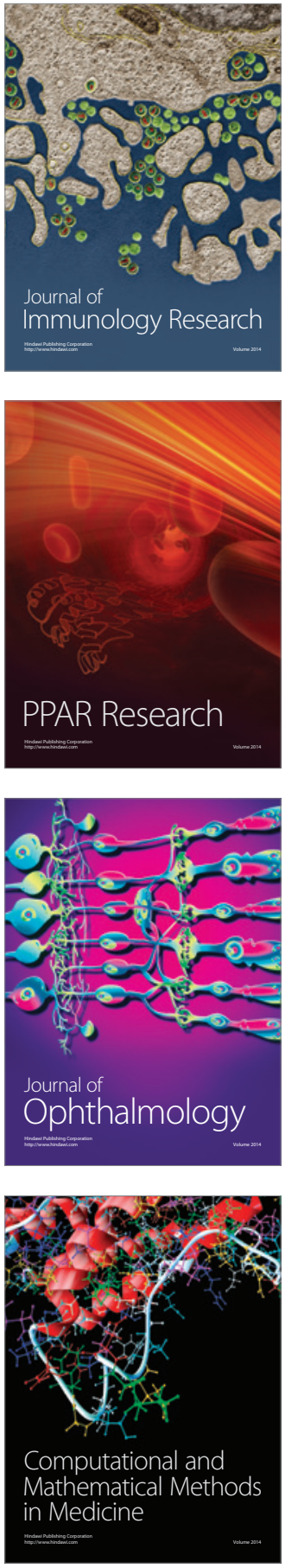

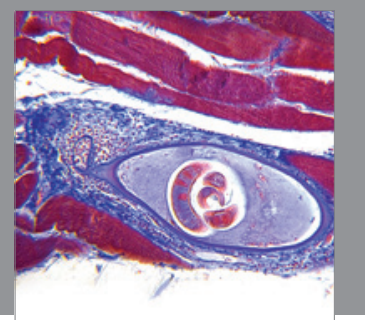

Gastroenterology

Research and Practice
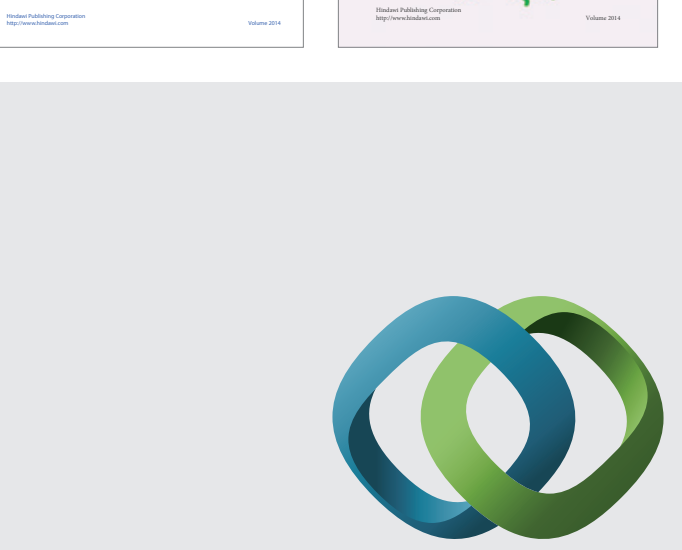

\section{Hindawi}

Submit your manuscripts at

http://www.hindawi.com
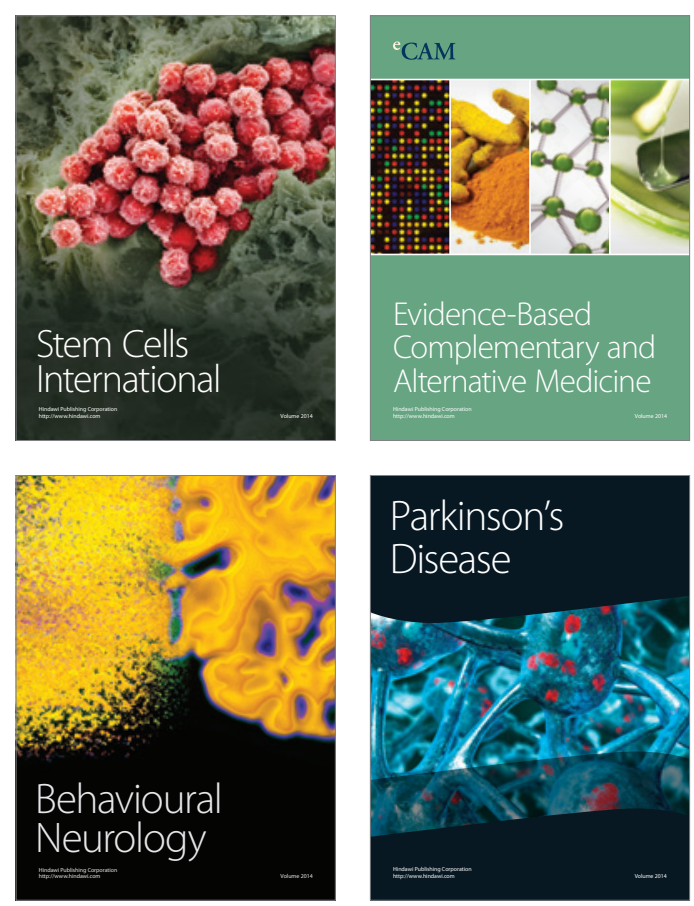

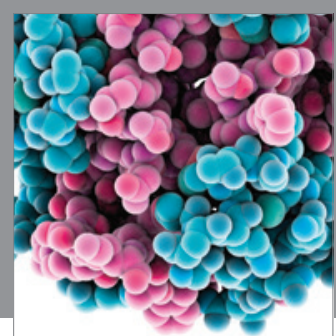

Journal of
Diabetes Research

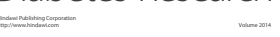

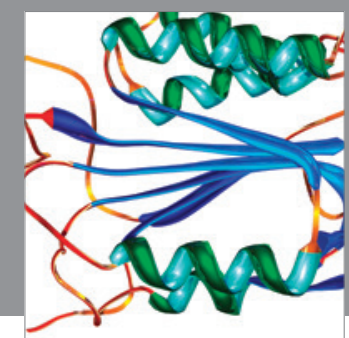

Disease Markers
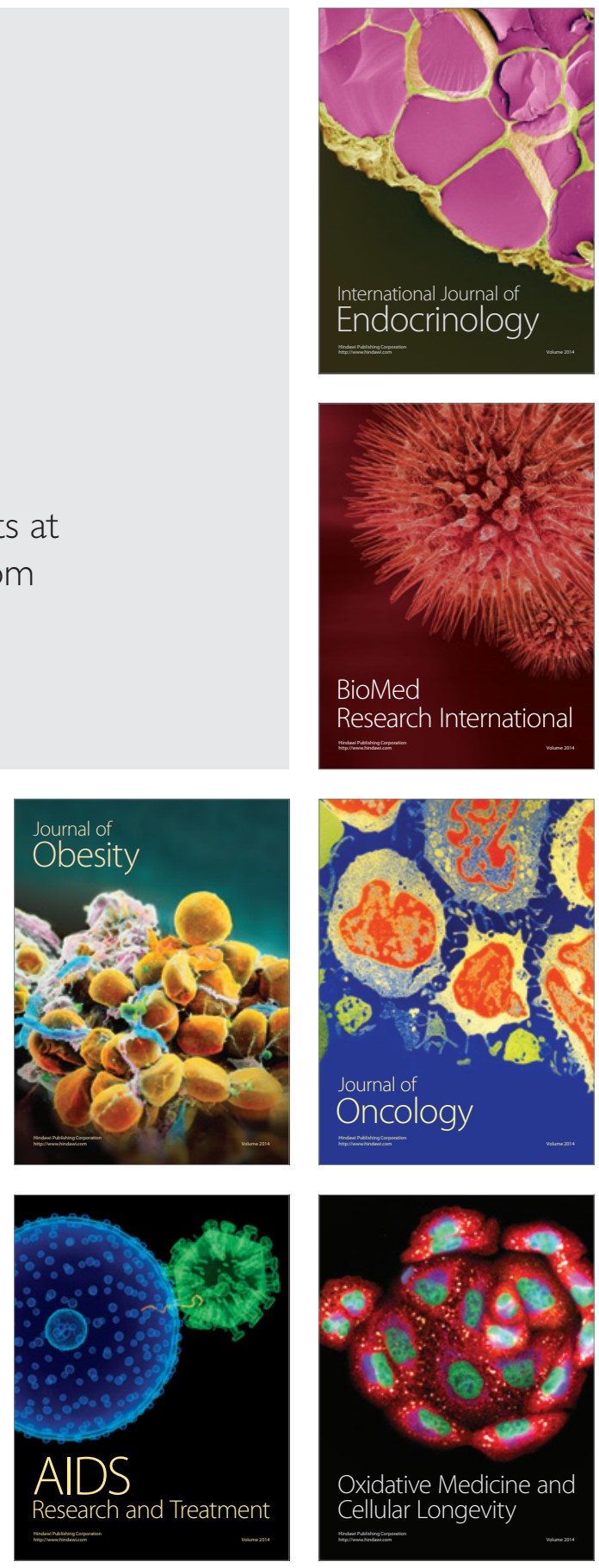